\title{
Accidental transdermal methanol poisoning presenting to a regional emergency department
}

\author{
Chelsea R. Beaton, MD*+ ; Clinton Meyer, BSc, MBBCh, MMed ${ }^{* \ddagger}$
}

Learning Points:

- Know and identify clinical presentations of toxic alcohols.

- Understand the differential diagnosis of high anion gap metabolic acidosis.

- Appreciate the importance of history and clinical findings in establishing methanol toxicity diagnoses, especially in centres where laboratory testing is unavailable.

- Recognize the value of provincial poison centres in supporting emergency physicians in the diagnosis and management of poisonings and overdoses.

Keywords: fomepizole, high anion gap metabolic acidosis, methanol, poisoning, toxicity, transdermal

\section{INTRODUCTION}

Toxic alcohols can be mistakenly ingested as an alternative to conventional ethanol or as an attempt at selfharm. Methanol, a central nervous system depressant and highly toxic alcohol, is commonly found in household and industrial products. ${ }^{1,2}$ Its metabolites, formaldehyde, and formic acid are responsible for the toxic effects observed following methanol intoxication. These effects may present only 12-24 hours after exposure and involve symptoms like blurred vision, scotomas, drowsiness, nausea, vomiting, abdominal pain, respiratory difficulties, unexplained seizures, coma, and cardiorespiratory arrest. ${ }^{1-5}$ Methanol poisoning occurs via ingestion, inhalation, or transdermal absorption, ${ }^{2}$ but case reports of the latter are rare. We report, to the best of our knowledge, the first case of accidental transdermal methanol exposure that resulted in significant toxicity in Canada.

\section{CASE REPORT}

A previously healthy 45-year-old male presented to our regional emergency department (ED) complaining of a 3-day history of epigastric pain, nausea, non-bloody emesis, and the inability to tolerate oral intake. The patient endorsed a 1-day history of lightheadedness, lethargy, shortness of breath, and vision changes. He denied any alcohol intake or illicit substance use. The patient recalled using a generic brand solvent to clean his hands and arms after staining his deck prior to the onset of symptoms and had not showered since the exposure. His only medication was omeprazole for Barrett's esophagus, and no significant past medical, allergy, or travel history were reported.

On examination, the patient appeared unwell, with the following vital signs: temperature $36.3^{\circ} \mathrm{C}$, blood pressure $143 / 91$, heart rate 98 , respiratory rate 20 , oxygen saturation $99 \%$ on room air. He was pale and diaphoretic with dry mucous membranes. On respiratory exam, hyperpnoea was noted. The abdominal exam revealed tenderness to the epigastrium on palpation with no guarding or rebound. The neurological exam revealed

From the *Battlefords Union Hospital, North Battleford, Saskatchewan; †North Battleford Rural Family Medicine Residency Program, University of Saskatchewan College of Medicine, North Battleford, Saskatchewan; and the ¥Department of Academic Emergency Medicine, University of Saskatchewan, Saskatoon, Saskatchewan.

Correspondence to: Dr. Chelsea R. Beaton, North Battleford Rural Family Medicine Residency Program, University of Saskatchewan College of Medicine, 102-11427 Railway Avenue, North Battleford, SK S9A 3G8; Email: crb749@mail.usask.ca

(C) Canadian Association of Emergency Physicians

CJEM 2019;21(3):435-437

DOI 10.1017/cem.2018.499 
decreased visual acuity, bilateral central scotomas, and colour deficits.

Laboratory testing showed a significantly elevated anion gap metabolic acidosis $(40.3 \mathrm{mmol} / \mathrm{L}$, venous $\mathrm{pH}$ $7.15)$, a low bicarbonate level $(6 \mathrm{mmol} / \mathrm{L})$, an acute $\mathrm{kid}-$ ney injury (creatinine $149 \mathrm{umol} / \mathrm{L}$, urea $12.9 \mathrm{mmol} / \mathrm{L})$, and negative ethanol and salicylate levels $(<3 \mathrm{mmol} / \mathrm{L}$, $<0.22 \mathrm{mmol} / \mathrm{L}$ ).

The history, clinical presentation, and laboratory findings raised our suspicion of methanol exposure. The history of solvent exposure was a confounding factor, prompting us to consult Poison and Drug Information Services (PADIS) and the medical toxicologist on call to confirm whether the solvent was a hydrocarbon and to determine whether exposure could result in a similar clinical presentation (considered unlikely). A presumptive diagnosis of methanol toxicity was made, despite the lack of exposure history. Unfortunately, our laboratory was unable to provide measured osmolality or methanol levels to confirm the diagnosis.

After suggesting possible methanol toxicity, the patient admitted that he may have confused methyl hydrate, a de-icer, with the solvent he kept on the same shelf.

As suggested by the medical toxicologist, we commenced a loading dose of fomepizole $(1.2 \mathrm{~g})$ and a sodium bicarbonate infusion. In doing so, we used the two ampules of fomepizole that our hospital carries at any given time. We then transferred the patient to a tertiary care centre for continued management. Subsequent laboratory values confirmed an elevated methanol level $(8 \mathrm{mmol} / \mathrm{L})$ and osmolar gap $(21 \mathrm{mOsm} / \mathrm{kg})$. The patient underwent hemodialysis and quickly improved. Sadly, his visual disturbances persisted, likely due to bilateral optic neuropathy.

\section{DISCUSSION}

Acute methanol toxicity typically occurs secondary to ingestion. Rarely have cases ensued from inhalation or transdermal absorption. ${ }^{2}$ Only a handful of cases describing dermal exposure are found in the literature, with none being reported in Canada. ${ }^{3,4}$

Patient presentations of methanol toxicity may vary; however, classic symptomologies like ocular toxicity (caused by formic acid inhibiting cytochrome oxidase and thus damaging the optic nerve's microtubule and mitochondrial components ${ }^{1,2,5}$ ) exist. The history of

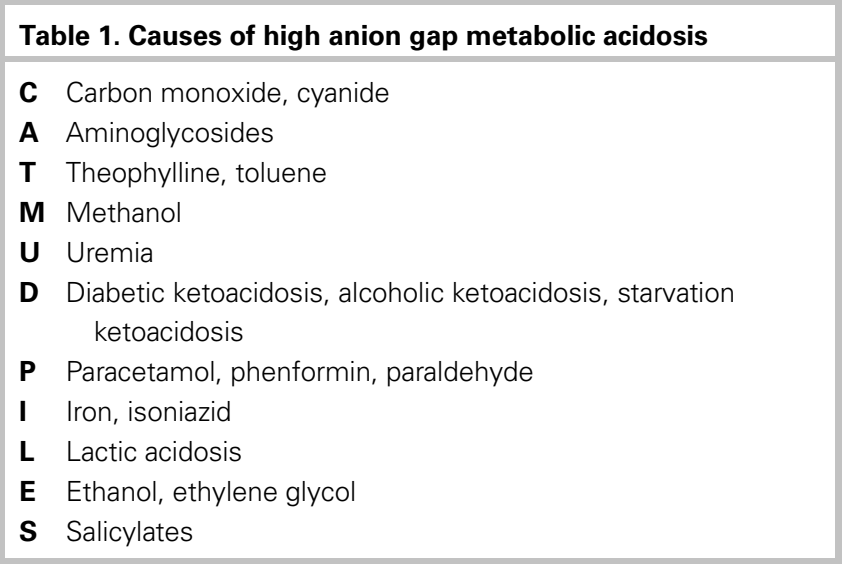

visual changes in our patient was crucial in raising suspicion and making a presumptive methanol toxicity diagnosis.

Methanol poisoning is confirmed by serum methanol levels and supported by anion and osmolar gap measurements. However, these tests are often not readily available in smaller centres across Canada, making this a challenging diagnosis. An additional diagnostic clue is a low serum bicarbonate $(<10 \mathrm{mmol} / \mathrm{L})$. As mentioned previously, toxic alcohol levels and serum osmolality were not available in our ED. Thus, after conferring with PADIS, we used our patient's high anion gap metabolic acidosis and low measured bicarbonate, which were not explained by other causes (Table 1), as an indication for empiric treatment for a likely methanol poisoning.

Other clinically significant toxic alcohols, like ethylene glycol and isopropanol, are also important to recognize. Patients with ethylene glycol toxicity may exhibit acute kidney failure, metabolic acidosis, hyperosmolality, hypocalcemia, and calcium oxalate crystals on urine microscopy. ${ }^{5}$ Conversely, those presenting with isopropanol poisoning can present with acetone breath, coma, hypotension, and hyperosmolality without acidosis. $^{5}$

It is imperative to rapidly identify and manage methanol toxicity to lessen the rate of morbidity and mortality in patients with significant exposure. ${ }^{1}$ The goal of treatment includes the use of fomepizole (15 $\mathrm{mg} / \mathrm{kg}$ IV loading dose) to inhibit alcohol dehydrogenase and prevent toxic metabolite formation. ${ }^{2,5}$ If unavailable, ethanol can be used (although off label). ${ }^{2,5}$ Acidosis correction with sodium bicarbonate is indicated when $\mathrm{pH}$ is $<7.3$. $^{5}$ The use of folic acid is postulated to modify deleterious metabolic pathways, and removal of toxins 
and metabolites by hemodialysis is considered in cases of severe toxicity. ${ }^{1,5}$ The early methanol toxicity diagnosis in our patient allowed for expedited treatment and an early transfer to a tertiary centre for diagnosis confirmation and definitive management.

\section{CONCLUSION}

This case highlights numerous pertinent clinical considerations for emergency physicians, especially in rural settings. In patients presenting with a high anion gap metabolic acidosis and physical exam findings without a supporting history, it is crucial to be highly suspicious of toxic alcohol poisoning and to contact local poison centres for support. It is also important to consider all routes of absorption. Furthermore, most smaller centres do not have access to confirmatory testing, thus it is imperative to use one's clinical gestalt to initiate treatment based on a presumptive diagnosis to reduce methanol's long-term toxic effects.
Competing interests: None declared.

Acknowledgements: The authors would like to thank Ms. Catherine Hana, the librarian for the Saskatchewan Health Authority, for her assistance with the literature review.

\section{REFERENCES}

1. Henderson W, Brubacher J. Methanol and ethylene glycol poisoning: a case study and review of current literature. CFEM 2002;4(1):34-40.

2. Barceloux D, Bond R, Krenzelok E, et al. American Academy of Clinical Toxicology practice guidelines on the treatment of methanol poisoning. Clin Toxicol 2002;40(4):415-46.

3. Uca A, Kozak H, Atlas M. An undercovered health threat in Turkey: transdermal methanol intoxication. Clin Neuropharmacol 2015;38(2):52-4.

4. Karaduman F, Asil T, Balci K, et al. Bilateral basal ganglionic lesions due to transdermal methanol intoxication. 7 Clin Neurosci 2009;16:1504-6.

5. Kraut J, Kurtz I. Toxic alcohol ingestions: clinical features, diagnosis, and management. Clin $7 \mathrm{Am}$ Soc Nephrol 2008;3:208-25. 